\title{
Cost and Utilization Outcomes After Exclusion of Dipeptidyl Peptidase-4 Inhibitors and 0ther Diabetes Drug Category Changes in a Self-Funded, State Employee Managed Care Plan
}

\author{
Jarrod King, PharmD; Carrie McAdam-Marx, MSCI, PhD, RPh; Rachael McCaleb, PharmD, BCPS; \\ Dwight Davis, PharmD; Geri Beth Bemberg, PharmD; and Jill T. Johnson, PharmD, BCPS
}

\begin{abstract}
BACKGROUND: Dipeptidyl peptidase-4 (DPP-4) inhibitors have repeatedly shown no reduction in the clinical outcomes of cardiovascular death, myocardial infarction, stroke, or all-cause mortality. Because the treatment of diabetes is generally one of the top drug categories by cost to health plans and self-funded employers, it is necessary to evaluate coverage of DPP-4 inhibitors, considering their lack of cardiovascular benefit relative to other treatment options.
\end{abstract}

OBJECTIVE: To describe the cost and utilization outcomes of drugs used to treat diabetes after exclusion of DPP-4 inhibitors in a self-funded managed care plan.

METHODS: This study was a retrospective, descriptive analysis of the cost and utilization outcomes after exclusion of DPP-4 inhibitors. Pharmacy claims data and plan membership were analyzed 6 months before DPP-4 inhibitor exclusion (preperiod: December 1, 2016-May 31, 2017) and 6 months after DPP-4 inhibitor coverage ended for all users (postperiod: September 1, 2017- February 28, 2018). The allowed amount, which is not influenced by overlapping plan copay changes, and utilization per member per month (PMPM) were used to estimate the effect of the DPP-4 inhibitor benefit exclusion on plan costs for the antidiabetic class.

RESULTS: From preperiod to postperiod, all DPP-4 inhibitor products decreased in utilization by 3.02 claims per 1,000 members per month (PTMPM). Glucagon-like peptide-1 receptor agonists, insulins, sodiumglucose cotransporter-2 inhibitors, and thiazolidinedione claims increased by $0.72,0.43,0.30$, and 0.48 claims PTMPM, respectively, but there was an absolute decrease of 1.35 claims for antidiabetic medications per 1,000 plan members. However, the days supplied PMPM increased from 2.55 to $2.61(2.3 \%)$ days. Allowed amount PMPM increased by $\$ 0.27$ from $\$ 12.19$ in the preperiod to $\$ 12.31$ in the postperiod $(2.2 \%)$. However, it is estimated that drug cost inflation accounted for over half of the PMPM increase in allowed costs.

CONCLUSIONS: The observed increase in the allowed amount PMPM was attributable in similar amounts by an increase in utilization of medications with higher cost per day supplied and higher drug prices. Future research will evaluate patient-level effects of this benefit change in terms of antidiabetic medication utilization and outcomes.

J Manag Care Spec Pharm. 2019;25(6):646-51

Copyright $\odot 2019$, Academy of Managed Care Pharmacy. All rights reserved.

\section{What is already known about this subject}

To date, for treatment of type 2 diabetes mellitus, only liraglutide and empagliflozin have received FDA approval for risk reduction of major cardiovascular events in a defined population.

Although well tolerated, dipeptidyl peptidase-4 (DPP-4) inhibitors have not shown cardiovascular risk reduction over placebo; some DPP-4 inhibiors increase heart failure risk.

Hemoglobin Alc reduction with DPP-4 inhibitors is similar to modestly less than with other oral agents

\section{What this study adds}

There was an increase in overall diabetes medication ingredient costs per member per month (PMPM) of $\$ 0.27$, or $4.0 \%$, after DPP-4 inhibitor exclusion.

Approximately half of the increase in allowed amount PMPM was driven by drug price inflation, with the other half likely due to a shift from DPP-4 inhibitors to medications with higher cost. Use of medications with proven cardiovascular benefit, such as thiazolidinediones, glucagon-like peptide-1 receptor agonists, and sodium-glucose cotransporter-2 inhibitors, increased after exclusion of DPP-4 inhibitors.

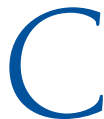
ardiovascular disease is the leading cause of death for patients with type 2 diabetes mellitus (T2DM). Several drugs used to treat T2DM have been shown to reduce the risk of microvascular or macrovascular events, while others have been shown to only improve surrogate endpoints such as hemoglobin Alc (Alc). Since 2008, the U.S Food and Drug Administration's (FDA) guidance has required large cardiovascular outcomes trials for all new T2DM drug therapies in order to protect the public health by ensuring safety and efficacy of new diabetes drugs, rather than to identify benefit. ${ }^{1}$ Correlation between tight blood glucose control and cardiovascular risk and mortality is unclear. ${ }^{2,3}$ Nevertheless, some of the drugs have managed to provide a statistically significant benefit by reducing cardiovascular events or mortality over placebo. ${ }^{4-7}$

The Arkansas State Employee Benefits Division (EBD) is a large, self-insured trust fund composed of state and public school employees, along with spouses and dependents. 
In 2017, the EBD covered approximately 150,000 beneficiaries, of which approximately 68,615 (46\%) were utilizing members. EBD plan members typically maintain employment as state employees or public school employees for years. As such, the EBD population is stable relative to commercial health plans. Given plan member longevity, the EBD is motivated to ensure that the pharmacy benefit is designed to optimize short- and long-term outcomes, even if changes result in medication cost increases. This is particularly relevant for diabetes, which is currently the second most costly drug category by plan-paid, with a plan spend of over $\$ 19$ million dollars per year. Given a goal of optimizing outcomes, the EBD prioritized diabetes drug coverage to agents that have been shown to reduce the risk of diabetes complications, either microvascular or macrovascular, and limit or exclude access to agents that have been shown to improve only intermediary outcomes such as Alc.

Under this decision framework, the EBD focused on the dipeptidyl-peptidase 4 (DPP-4) inhibitor class of drugs, which comprised $4.4 \%$ of all diabetes utilization and $9.7 \%$ of all the diabetes drug plan-paid. The plan recognized that DPP-4 inhibitor monotherapy is well tolerated, weight neutral, and does not carry a risk of causing hypoglycemia. However, available evidence has shown that it does not reduce the risk of major cardiovascular events in any prospective, randomized controlled trial better than placebo. ${ }^{8-12}$ There is also a lack of evidence of microvascular risk reduction for DPP-4 inhibitors. Thus, the EBD decided to end coverage of DDP-4 inhibitors on June 1, 2017. This study describes the changes in antidiabetic drug use and ingredient cost before and after implementing the exclusion of DPP-4 inhibitors.

During the study period, no incentive was provided to drive members to 90-day supplies, and no changes in dispensing fees occurred. Other changes were made to diabetes drug coverage at the same time. Notably, liraglutide was moved from tier 3 to tier 2 and designated as the exclusive glucagon-like peptide receptor agonist (GLP-1 RA). Empagliflozin was selected as the exclusive sodium-glucose cotransporter-2 (SGLT2) inhibitor and was moved from tier 3 to tier 2, and U-500 human insulin was moved from tier 2 to tier 3 .

\section{Methods}

A pre-post, descriptive analysis based on aggregated pharmacy claims data was performed to assess changes in cost and utilization before and after the DPP-4 inhibitor benefit exclusion. The data did not contain patient identifiable data, was HIPAA compliant, and did not require institutional review board approval, since the study was not human subjects research. Statistical analysis was not performed because of a relatively limited number of months available after exclusion of DPP-4 inhibitors.

Claims for antidiabetic medications were obtained from the EBD pharmacy claims database using the First Data Bank Generic Therapeutic Class (GTC) code "DIABETES" for claims with dates of service from December 1, 2016, to February 28, 2018. Diabetic drug classes included products containing DPP-4 inhibitors, insulins, thiazolidinediones (TZDs), sulfonylureas, biguanides, SGLT2 inhibitors, GLP-1 RAs, all other combination products, and all other products.

The data consisted of total plan enrollment count and aggregated pharmacy claims data by drug class for the 6 months before exclusion of DPP-4 inhibitors from new users (preperiod: December 1, 2016, through May 31, 2017), for the 90-day grace period for current users (transition period: June 1, 2017, through August 31, 2017), and for the 6 months after exclusion of all users from DPP-4 inhibitors (postperiod: September 1, 2017, through February 28, 2018). While aggregate population demographic characteristics were not reported, the eligible population remained relatively constant during the study evaluation period.

Antidiabetic drug cost and utilization information was obtained from the pharmacy claims data for each study period and included number of prescriptions, days supply, and ingredient cost. Copay amount was not included due to changes in copays for select antidiabetic agents, as previously described. Thus, we do not report amount paid by the plan net copay, since that value reflects the DPP-4 benefit exclusion and concurrent copay changes. Ingredient cost reflects the change in costs driven by shifts in utilization rather than copay changes.

Cost and utilization data were normalized to per member per month (PMPM) to account for enrollment changes, and changes in cost and utilization between the preperiod and postperiod were identified. Utilization by antidiabetic drug class was also calculated per 1,000 members per month (PTMPM).

To isolate inflation from utilization change as a driver of cost, the preperiod ingredient cost per day of therapy was identified by class. The average preperiod allowed amount per day was multiplied by postperiod days supply to estimate the postperiod allowed amount had there been no drug price changes.

\section{Results}

\section{Diabetes Drug Class Shifts in Utilization and Market Share}

Utilization changes by drug class over the study period are presented in Figure 1 for all classes except biguanides, insulin, and sulfonylureas due to their high overall use. There were 3.15 DPP-4 inhibitor claims PTMPM during the preperiod. Following the formulary exclusion of DPP-4 inhibitors, there were 0.14 claims PTMPM, a decrease of 3.01 claims PTMPM. Overall, this was associated with a $95.7 \%$ reduction in claims for DPP-4 inhibitors. Market share, as measured by the percentage of all antidiabetic drug claims, was $4.4 \%$ for DPP-4 inhibitors in the preperiod and $0.2 \%$ in the postperiod.

Utilization of other antidiabetic drug classes increased after the DPP-4 inhibitor coverage exclusion, with utilization increase greatest in the GLP-1 RA class. GLP-1 RA had 1.56 
Cost and Utilization Outcomes After Exclusion of Dipeptidyl Peptidase-4 Inhibitors and Other Diabetes Drug Category Changes in a Self-Funded, State Employee Managed Care Plan

\section{FIGURE 1 Select Antidiabetic Drug Utilization by Month per 1,000 Plan Memebers ${ }^{\mathrm{a}}$}

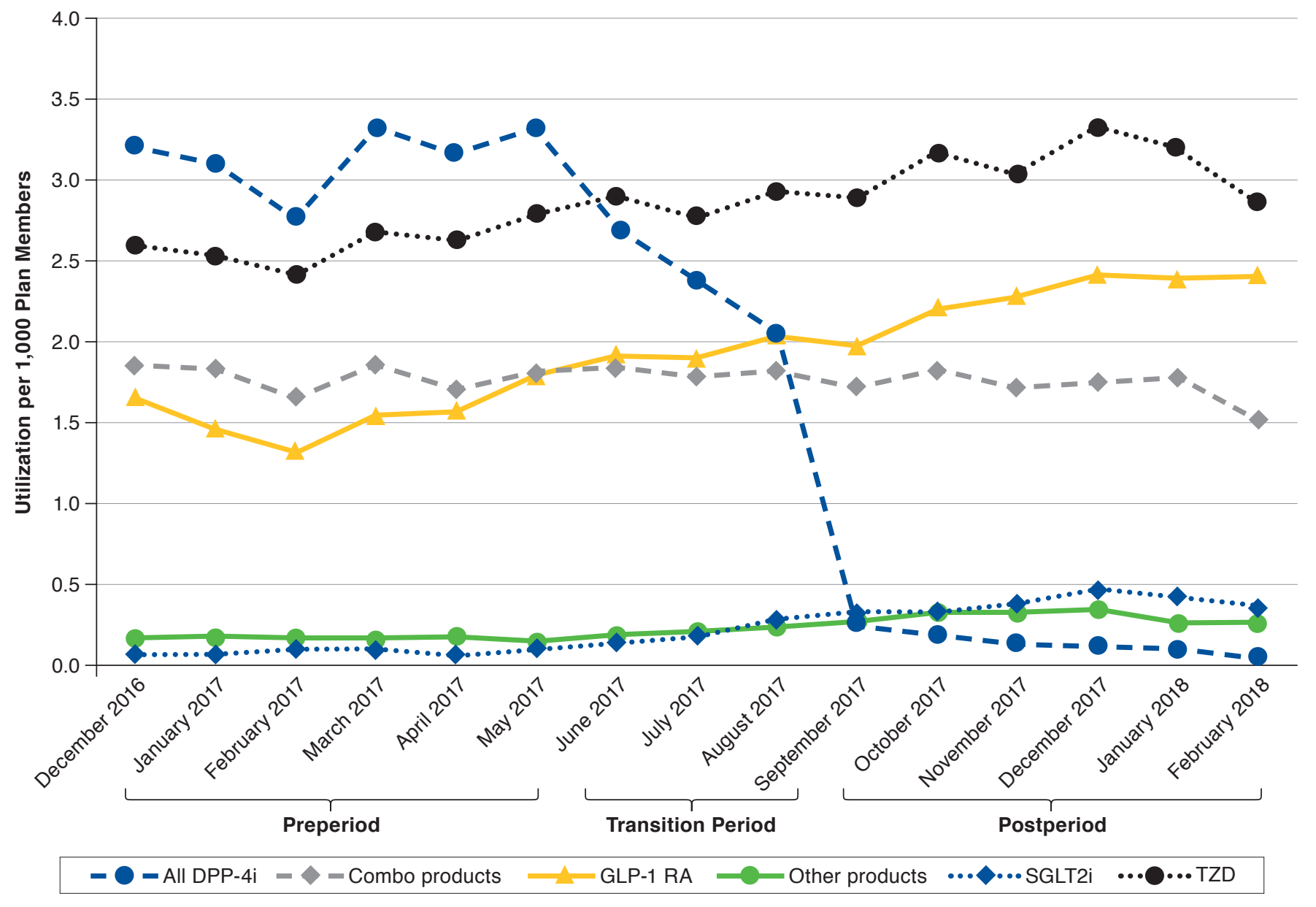

aDue to higher utilization, metformin, insulin, and sulfonylureas are not included in this figure.

DPP-4i=dipeptidyl peptidase-4 inhibitor; GLP-1 RA=glucagon-like peptide receptor agonist; SGLT2i=sodium-glucose cotransporter-2 inhibitor; TZD=thiazolidinediones .

claims PTMPM in the preperiod and 2.28 claims PTMPM in the postperiod, an increase of 0.72 claims PTMPM, or $46.4 \%$. Market share of GLP-1 RA increased from 2.2\% in the preperiod to $3.3 \%$ in the postperiod. TZDs had the second highest absolute increase in claims PTMPM at 0.48. There were 2.61 claims PTMPM in the preperiod and 3.08 in the postperiod. There was also an absolute increase in market share of $0.8 \%$, from $3.7 \%$ in the preperiod to $4.4 \%$ in the postperiod. SGLT2 inhibitor use was small in the preperiod and postperiod, with claims PTMPM increasing from 0.08 to 0.38 PTMPM from the preperiod to postperiod, respectively. There was also an increase in market share from $0.1 \%$ to $0.5 \%$.

Biguanides were the most dispensed product at $45.7 \%$ of the market share. There was a slight decrease in use, from 32.3 claims PTMPM in the preperiod to 32.0 in the postperiod.
Insulins were consistently the second most dispensed antidiabetic drug PMPM but had the third highest absolute increase in claims PTMPM behind GLP-1 RA and TZDs. There were 14.8 claims PTMPM in the preperiod and 15.3 in the postperiod, a $2.9 \%$ increase. Market share of insulins increased by $1.0 \%$, from $20.9 \%$ to $21.9 \%$ of all antidiabetic claims.

\section{Plan Membership, Cost, and Utilization of Antidiabetic Drugs Before and After Exclusion of DPP-4 Inhibitors}

Membership, use, and cost results are presented in Table 1 . The average monthly membership in the plan was 150,007 during the preperiod and 152,692 during the postperiod (+1.8\%). There was an absolute decrease in the diabetic medication claims per month, from 10,654 in the preperiod to 10,638 in the postperiod $(-0.2 \%)$, but an increase in days supplied from 
TABLE 1 Summary of Cost and Utilization of Antidiabetic Drugs Before and After Exclusion of DPP-4 Inhibitors

\begin{tabular}{|c|c|c|c|c|c|c|}
\hline & Member Months & Claims & Days Supply & Days Per Claim & $\begin{array}{l}\text { Total Allowed } \\
\text { Cost, } \$\end{array}$ & $\begin{array}{l}\text { Allowed Cost } \\
\text { PMPM, \$ }\end{array}$ \\
\hline \multicolumn{7}{|c|}{ Preperiod: Coverage of DPP-4 inhibitors } \\
\hline Total & 900,044 & 63,921 & $2,298,664$ & & $10,975,044$ & \\
\hline Average per month & 150,007 & 10,654 & 383,111 & 35.96 & $1,829,174$ & 12.19 \\
\hline \multicolumn{7}{|c|}{ Postperiod: Exclusion of DPP-4 inhibitors } \\
\hline Total & 916,151 & 63,825 & $2,393,606$ & & $11,418,832$ & \\
\hline Average per month & 152,692 & 10,638 & 398,934 & 37.50 & $1,903,139$ & 12.46 \\
\hline $\begin{array}{l}\text { Change per month from } \\
\text { preperiod }\end{array}$ & 2,685 & -16 & 15,824 & 1.54 & 73,965 & 0.27 \\
\hline Change from preperiod, $\%$ & 1.8 & -0.2 & 4.1 & 4.3 & 4.0 & 2.2 \\
\hline \multicolumn{7}{|c|}{ Postperiod: Based on preperiod costs per day to isolate utilization change } \\
\hline Total & & & & & $11,277,763$ & \\
\hline Average per month & & & & & $1,879,627$ & 12.31 \\
\hline $\begin{array}{l}\text { Estimated change per month } \\
\text { due to utilization }\end{array}$ & & & & & 50,453 & 0.12 \\
\hline $\begin{array}{l}\text { Estimated change due to } \\
\text { utilization, \% }\end{array}$ & & & & & 2.8 & 1.0 \\
\hline
\end{tabular}

383,111 in the preperiod to $398,934(+4.1 \%)$ in the postperiod. Days supply per claim averaged 35.96 in the preperiod to $37.50(+4.3 \%)$ in the postperiod. Allowed amount cost increased $4.0 \%$, from $\$ 1,829,174$ per month in the preperiod to $\$ 1,903,139$ per month in the postperiod, an increase of $\$ 73,965$ per month.

Total claims for the antidiabetic drugs class decreased from an average of 0.071 PMPM in the preperiod to 0.070 in the postperiod (-1.9\%; data not shown). The allowed amount PMPM was $\$ 12.19$ in the preperiod and $\$ 12.46$ during the postperiod, an increase of $\$ 0.27$, or $+2.2 \%$.

\section{Analysis of Intervention Effect and Drivers}

In an attempt to isolate utilization shifts from drug price changes, we held ingredient price constant by multiplying the days supply in the postperiod for each diabetes drug class by the preperiod allowed amount per day for that class. The overall estimated allowed amount increase due to changes in utilization was $\$ 50,453$ of the increase per month. When normalizing for enrollment, PMPM in the postperiod based on preperiod drug costs was $\$ 12.31$, or $\$ 0.12$ of the difference and a $1 \%$ increase from the preperiod.

\section{Discussion}

This retrospective, descriptive study evaluated the economic impact of a self-funded managed care plan's decision to remove DPP-4 inhibitors from its formulary and end benefit coverage of all DPP-4 inhibitors after a 90-day transition period for existing users. This action was associated with a 95\% reduction in the use of DPP-4 inhibitors across the health plan.
There remained a small utilization of DPP-4 inhibitor products because of an exception process within the plan. There was also a corresponding increase in the use of other diabetic drug classes and an increase in antidiabetic prescription allowed amount of $4.0 \%$ per month and \$0.27 PMPM (2.2\% increase).

The benefit change reflected a value-based formulary decision. While DPP-4 inhibitors are well tolerated and weight neutral, their efficacy is comparable with SGLT2 inhibitors and less effective than GLP-1 RAs in improving glycemic control. ${ }^{13-15}$ Recent cardiovascular outcomes trials for liraglutide (LEADER) and semaglutide (SUSTAIN-6) have shown cardiovascular benefit, ${ }^{4,6}$ and a real-world study also identified that liraglutide was associated with reduced cardiovascular risk and all-cause mortality relative to DPP-4 inhibitors. ${ }^{16}$ SGLT2 inhibitor cardiovascular outcomes trials for empagliflozin (EMPA-REG) and canagliflozin (CANVAS) have demonstrated cardiovascular and renal benefit., ${ }^{5,717}$ The 4 completed cardiovascular outcomes trials for saxaglitin, alogliptin, sitagliptin, and linagliptin (SAVOR-TIMI 32, EXAMINE, TECOS, and CAROLINA, respectively) have shown that while DPP-4 inhibitors are noninferior to placebo for cardiovascular outcomes, they did not demonstrate a cardiovascular benefit. ${ }^{8-12}$ Rather, there is some evidence that DPP-4 inhibitors, specifically sitagliptin and alogliptin, may increase risk of heart failure. ${ }^{16}$ While GLP-1RAs and SGLT2 inhibitors are more expensive options, the health plan decided to exclude DPP-4 inhibitors from the formulary.

After excluding DPP-4 inhibitors from coverage, use of SGLT2 inhibitors and GLP-1 RAs increased, as did the use of TZDs and insulin. With this utilization shift, allowed amount PMPM for diabetes medications increased 2.2\%. Some of this 


\section{Cost and Utilization Outcomes After Exclusion of DipeptidyI Peptidase-4 Inhibitors and Other Diabetes Drug Category Changes in a Self-Funded, State Employee Managed Care Plan}

increase represents a shift to more expensive agents. However, we estimate that over half of the observed allowed amount increase was due to an increase in price of diabetes drugs between the baseline and follow-up periods.

\section{Limitations}

There are several limitations of this descriptive analysis that should be taken into consideration when interpreting results. This study was observational in nature and did not include a comparison group not subject to the benefit change. The cohort was based on a single employer-sponsored health plan, which provided a stable cohort of patients for a pre-post analyses, but results may not be applicable to broader commercial, Medicare, or Medicaid plans.

The analysis, as an initial benefit change evaluation, was conducted at the population versus patient level. Thus, it would not be appropriate to draw conclusions from this study about the effects of this benefit change on individual patients, including the proportion of patients who may have discontinued a DPP-4 inhibitor without initiating other antidiabetic therapy. In addition, this study was limited to prescription drug data and was therefore not a comprehensive evaluation of the utilization and cost impact of the benefit change. Further, costs to the plan in communicating the benefit change to patients and providers and handling exception requests were not included nor were plan and patient office visit costs related to the benefit change. Manufacturer rebate data, which are proprietary, were also not included. Future evaluation of this benefit change will be conducted at the patient level to assess individual patient switching patterns, medical and benefit administration costs, and outcomes. Patient-level data will also allow us to control for disease severity.

Finally, because there were several other benefit changes that occurred at the same time as the DPP-4 inhibitor exclusion, including copay changes for liraglutide, empagliflozin, and Humulin 500 unit vials, we reported costs based on allowed amount rather than plan cost net copay. While market share of these products is relatively limited, copay changes, which were largely reductions, would cloud the effect of DPP-4 inhibitors on plan costs. Thus, health plans must consider their copay/ coinsurance structures and rebate agreements when attempting to extrapolate these findings to their unique settings.

\section{Conclusions}

After exclusion of DPP-4 inhibitors, there was an increase in use of SGLT2 inhibitors, TZDs, and GLP-1 RAs, which all have proven cardiovascular benefit. These utilization changes were associated with an increase in allowed amount PMPM of $\$ 0.27$, a $2.2 \%$ increase, of which over half is estimated to be due to changes in drug prices versus utilization shifts. Future research will evaluate patient-level effects of this benefit change in terms of antidiabetic medication utilization and outcomes.

\section{Authors}

JARROD KING, PharmD; CARRIE MCADAM-MARX, MSCI, $\mathrm{PhD}, \mathrm{RPh}$; RACHAEL MCCALEB, PharmD, BCPS, University of Arkansas for Medical Sciences College of Pharmacy, Little Rock. DWIGHT DAVIS, PharmD, and JILL T. JOHNSON, PharmD, BCPS, Evidence-Based Prescription Drug Program, University of Arkansas for Medical Sciences College of Pharmacy, Little Rock. GERI BETH BEMBERG, PharmD, Stephens Insurance, Little Rock, Arkansas.

AUTHOR CORRESPONDENCE: Jill T. Johnson, PharmD, BCPS, Evidence-Based Prescription Drug Program, University of Arkansas for Medical Sciences College of Pharmacy, 4301 W. Markham, \#522-4, Little Rock, AR 72205. Tel.: 501.686.7919; E-mail: johnsonjill@@uams.edu.

\section{DISCLOSURES}

No outside funding supported this study. Davis, Bemberg, and Johnson currently work for or previously worked for the UAMS Evidence-Based Prescription Drug Program, which advises the Employee Benefits Division (EBD) on pharmacy benefit management. The EBD did not provide any additional funding for this study. McAdam-Marx reports grants from AstraZeneca and Sanofi Aventis outside the submitted work. The other authors have no other relevant information to disclose.

\section{ACKNOWLEDGMENTS}

The authors thank MedImpact for providing data reports for this analysis. The authors also acknowledge Chris Howlett, executive director of the EBD and nonvoting member of the Drug Use Evaluation Committee (a subcommittee of the Arkansas Insurance Board), who worked to administer the pharmacy benefit.

\section{REFERENCES}

1. Regier EE, Venkat MV, Close KL. More than 7 years of hindsight: revisiting the FDA's 2008 guidance on cardiovascular outcomes trials for type 2 diabetes medications. Clin Diabetes. 2016;34(4):173-80

2. Action to Control Cardiovascular Risk in Diabetes Study Group, Gerstein HC, Miller ME, Byington RP, et al. Effects of intensive glucose lowering in type 2 diabetes. N Engl J Med. 2008;358(24):2545-59.

3. ADVANCE Collaborative Group, Patel A, MacMahon S, Chalmers J, et al Intensive blood glucose control and vascular outcomes in patients with type 2 diabetes. N Engl J Med. 2008;358(24):2560-72

4. Marso SP, Daniels GH, Brown-Frandsen K, et al. Liraglutide and cardiovascular outcomes in type 2 diabetes. N Engl J Med. 2016;375(4):311-22.

5. Zinman B, Wanner C, Lachin JM, et al. Empagliflozin, cardiovascular outcomes, and mortality in type 2 diabetes. N Engl J Med. 2015;373(22): $2117-28$

6. Marso SP, Bain SC, Consoli A, et al. Semaglutide and cardiovascular outcomes in patients with type 2 diabetes. N Engl J Med. 2016;375(19):1834-44.

7. Neal B, Perkovic V, Mahaffey KW, et al. Canagliflozin and cardiovascular and renal events in type 2 diabetes. N Engl J Med. 2017;377(7):644-57.

8. Scirica BM, Bhatt DL, Braunwald E, et al. Saxagliptin and cardiovascular outcomes in patients with type 2 diabetes mellitus. N Engl J Med. 2013;369(14):1317-26. 
9. Zannad F, Cannon CP, Cushman WC, et al. Heart failure and mortality outcomes in patients with type 2 diabetes taking alogliptin versus placebo in EXAMINE: a multicentre, randomised, double-blind trial. Lancet. 2015;385(9982):2067-76.

10. Green JB, Bethel MA, Armstrong PW, et al. Effect of sitagliptin on cardiovascular outcomes in type 2 diabetes. N Engl J Med. 2015;373(3):232-42.

11. White WB, Cannon CP, Heller SR, et al. Alogliptin after acute coronary syndrome in patients with type 2 diabetes. N Engl J Med. 2013;369(14):1327-35.

12. Gallwitz B, Rosenstock J, Rauch T, et al. 2-year efficacy and safety of linagliptin compared with glimepiride in patients with type 2 diabetes inadequately controlled on metformin: a randomised, double-blind, noninferiority trial. Lancet. 2012;380(9840):475-83.

13. Roden M, Weng J, Eilbracht J, et al. Empagliflozin monotherapy with sitagliptin as an active comparator in patients with type 2 diabetes: a randomised, double-blind, placebo-controlled, phase 3 trial. Lancet Diabetes Endocrinol. 2013;1(3):208-19.
14. Ferrannini E, Berk A, Hantel S, et al. Long-term safety and efficacy of empagliflozin, sitagliptin, and metformin: an active-controlled, parallelgroup, randomized, 78-week open-label extension study in patients with type 2 diabetes. Diabetes Care. 2013;36(12):4015-21.

15. Hemmingsen B, Sonne DP, Metzendorf MI, Richter B. Dipeptidylpeptidase (DPP)-4 inhibitors and glucagon-like peptide (GLP)-1 analogues for prevention or delay of type 2 diabetes mellitus and its associated complications in people at increased risk for the development of type 2 diabetes mellitus. Cochrane Database Syst Rev. 2017;5:CD012204.

16. Svanström H, Ueda P, Melbye M, et al. Use of liraglutide and risk of major cardiovascular events: a register-based cohort study in Denmark and Sweden. Lancet Diabetes Endocrinol. 2019;7(2):106-14.

17. Cefalu WT, Kaul S, Gerstein HC, et al. Cardiovascular outcomes trials in type 2 diabetes: where do we go from here? Reflections from a Diabetes Care editors' Expert Forum. Diabetes Care. 2018;41(1):14-31. 